\title{
The future of risk stratification in thoracic surgery
}

\author{
Ioannis K. Toumpoulis, MD, ${ }^{a}$ Chris K. Rokkas, MD, ${ }^{b}$ and Themistocles P. Chamogeorgakis, MD
}

From the Department of Cardiothoracic Surgery, Beth Israel Deaconess Medical Center, Harvard Medical School, Boston, Mass ${ }^{\mathrm{a}}$ and the Department of Cardiothoracic Surgery, Attikon Hospital Center, University of Athens Medical School, Athens, Greece. ${ }^{\mathrm{b}}$

Received for publication March 2, 2008; accepted for publication March 12, 2008.

Address for reprints: Ioannis K. Toumpoulis, MD, Department of Cardiothoracic Surgery, Beth Israel Deaconess Medical Center, Harvard Medical School, 77 Ave Louis Pasteur, Room 144, Boston, MA 02115 (E-mail: toumpoul@otenet.gr).

J Thorac Cardiovasc Surg 2008;136:7-9

$0022-5223 / \$ 34.00$

Copyright $(02008$ by The American Association for Thoracic Surgery

doi:10.1016/j.jtcvs.2008.03.023
$\mathrm{R}$ isk stratification plays an important role in surgical specialties worldwide. Multivariable models are used to assess the clinical outcomes in an objective risk-adjusted manner and allow useful comparisons to be made between countries, regions, hospitals, and individual surgeons. The Society of Thoracic Surgeons (STS) Adult Cardiac Surgery Database in the United States has been one of the biggest and most reliable risk-adjusted databases, and it is the gold standard for clinical data analysis in the field of cardiac surgery. Similarly, the open source algorithm of the European System for Cardiac Operative Risk Evaluation (EuroSCORE), which was developed in Europe, represents another reliable, widely established and validated model for risk stratification in the entire context of cardiac surgery.

During the past 15 years, while STS and EuroSCORE algorithms were developed, thoracic surgery is lacking an accepted general model for in-hospital mortality. Recently, Thoracoscore became available, and it is the first multivariate model for the prediction of in-hospital mortality in the entire context of thoracic surgery. ${ }^{1}$ Thoracoscore was developed from 15,183 patients who underwent thoracic surgery in 59 French hospitals. In the meanwhile, the corresponding General Thoracic Database of the STS is still under development, accounting for 49,000 patients (1999-2006). However, there is no risk stratification model published to date from this North American database. All the aforementioned indicate that the risk stratification in thoracic surgery is in its birth and early stages. There are many reasons for general thoracic surgeons to help in the progression and the development of risk stratification in thoracic surgery, such as quality improvements in patient care and patient outcomes, differentiation from surgeons who are less qualified to perform thoracic surgery, recertification of surgeons, costs of surgery, and pay for performance. ${ }^{2}$

Thoracoscore is a reliable risk stratification model because it comes from a national electronic prospective database and therefore has the potential to reach an international consensus. Our research team has published the first study evaluating the performance of Thoracoscore in an external database. ${ }^{3}$ The conclusions of our study are supportive and encouraging. First, we evaluated the performance of Thoracoscore in a North American single-center cohort of patients with the entire context of thoracic surgery, and it showed excellent discriminatory ability and calibration in predicting in-hospital mortality. Second, we extended the usefulness of Thoracoscore with respect to midterm all-cause mortality prediction. Indeed, Thoracoscore showed very good discriminatory ability in predicting 2-year survival after thoracic surgery. Although in our study one of the original variables of the Thoracoscore was not available (dyspnea score), we concluded that it is a good clinical tool for preoperative prediction of inhospital and midterm mortality among patients undergoing general thoracic surgery.

Given the fact that Thoracoscore is the first step of risk stratification in thoracic surgery, the question regarding the future of risk stratification in this surgical field needs to be addressed to determine the next steps. A good example to reach an international consensus is the EuroSCORE development. EuroSCORE was first introduced in 1999, and there are 323 publications to date in the English language (March 2008) validating its algorithm throughout Europe, North America, and Asia. In addition, EuroSCORE has been proved to be a good predictor for major postoperative complications, costs, midterm survival, and long-term survival after cardiac surgery. ${ }^{4-7}$ Therefore, the evaluation of these outcomes in thoracic surgery by Thoracoscore or any other new score may help in the establishment of a useful multipurpose clinical tool. 


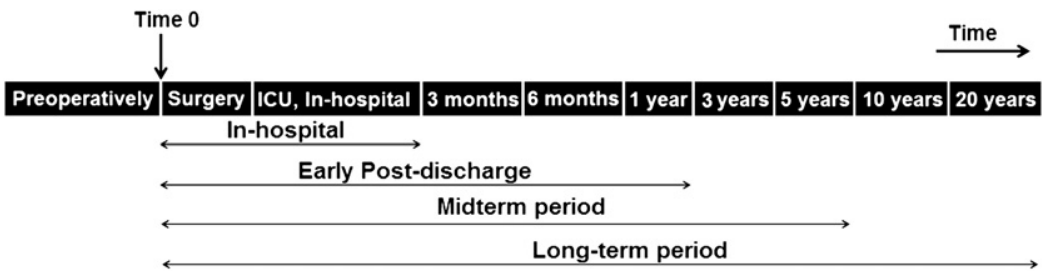

Figure 1. Time intervals after thoracic surgery. ICU, Intensive care unit.

At present, most surgical registries monitor patients only to the point of discharge from the hospital. However, it is widely known that in-hospital adverse events represent only one aspect of the periprocedural outcome. It is important to mention that the STS National Database aims to expand its capabilities to perform long-term follow-up using the National Death Index. Unique patient, surgeon, and hospital identifier fields have already been added to the STS Adult Cardiac Surgery Database beginning in January 2008, and similar fields will be added to the STS General Thoracic Surgery Database. The sensitivity of the National Death Index to identify deaths is between $92 \%$ and $99 \%$ depending on which identifiers are available. Social Security number alone has the best accuracy of any combination of other identifiers with a sensitivity of $97 \%$ and a specificity of $99 \% .^{8}$ The use of Social Security numbers in the STS databases will enable them to generate Kaplan-Meier survival curves, as well as to perform multivariate Cox regression analysis for long-term outcomes. The National Death Index can provide rapid and objective long-term survival results, particularly when used with specific software such as we have developed, with which thousands of queries can be obtained accurately in minutes. ${ }^{9}$

Figure 1 determines different time intervals after surgery. In-hospital stay is the target period for most stratification systems in cardiothoracic surgery. Early postdischarge period (first months after discharge up to 1 year after discharge) represents a very important period in risk stratification, because adverse events and complications directly related to the surgical procedure may also occur during this time interval. Furthermore, the midterm period (up to 5 years) could be an ideal time interval to extract conclusive and safe results for the outcome of a procedure. Finally, long-term follow-up (10 years or more) is needed in some cases to determine the efficacy of the procedure as well as to compare the outcome with the general population. Therefore, future risk stratification models in thoracic surgery should take into consideration midterm and long-term outcomes.

The next important step is to determine the outcome. Most risk stratification models in cardiothoracic surgery have been developed for the prediction of in-hospital mortality. Refinements in surgical techniques and improvements in anesthetic management and intensive care unit monitoring have resulted in reduced operative mortality. In this aspect, the development of risk stratification models for the prediction of in-hospital mortality requires the analysis of relatively large patient populations. On the other hand, major postoperative complications are also important and affect both in-hospital and midterm survival. ${ }^{7,10}$ Therefore, the prediction of major postoperative complications could help in improving patient selection for surgery, stratifying patients, and enhancing informed consent, as well as assigning resources for postoperative management. Another important issue is to demarcate disease or surgery-specific mortality from all-cause mortality. For inhospital mortality it may be easy to document the cause of death, but for long-term mortality there is no source to determine the cause of death, in as much as the National Death Index can provide data only for the survival status and the date of death. The characterization of disease-specific mortality during midterm and long-term follow-up periods remains unsolved and should be addressed by large databases to enhance them with the ability to provide more accurate long-term results based only on the analysis of the disease-specific outcome. Another important adverse event is the recurrence of the disease during the midterm or long-term period. It seems difficult to assess this outcome given the fact that usually the patient is been followed up by other specialists (ie, oncologists or family physicians). Finally, one of the most important outcomes for the patient is the quality of life after surgery. Even in cardiac surgery there is a paucity of risk stratification models with respect to quality of life after surgery. Such models are very important because the purpose of surgery is not only to ensure survival of the patient, but also to allow for a quality of life that is similar to or close to that of the general population. Table 1 summarizes these outcomes. All-cause and disease-specific mortality can be assessed at all time intervals,

TABLE 1. Different types of adverse events and outcome after thoracic surgery

\begin{tabular}{lcccc}
\hline \multicolumn{1}{c}{ Outcome } & In-hospital & $\begin{array}{c}\text { Early after } \\
\text { discharge }\end{array}$ & $\begin{array}{c}\text { Midterm } \\
\text { period }\end{array}$ & $\begin{array}{c}\text { Long-term } \\
\text { period }\end{array}$ \\
\hline $\begin{array}{l}\text { All-cause mortality } \\
\begin{array}{l}\text { Disease-specific } \\
\text { mortality }\end{array}\end{array}$ & $\sqrt{ }$ & $\sqrt{ }$ & $\sqrt{ }$ & $\sqrt{ }$ \\
$\begin{array}{l}\text { Postoperative } \\
\text { complications }\end{array}$ & $\sqrt{ }$ & $\sqrt{ }$ & $\sqrt{ }$ & $\sqrt{ }$ \\
$\begin{array}{l}\text { Disease recurrence } \\
\text { Quality of life }\end{array}$ & - & $\sqrt{ }$ & - & - \\
\hline
\end{tabular}


TABLE 2. Potential risk factors affecting outcomes after thoracic surgery

\begin{tabular}{|c|c|c|c|}
\hline \multicolumn{2}{|c|}{ Preoperatively known or unknown } & \multirow{2}{*}{$\begin{array}{c}\text { Occur intraoperatively } \\
\text { Intraoperative risk factors }\end{array}$} & \multirow{2}{*}{$\begin{array}{l}\text { Occur postoperatively } \\
\text { Follow-up risk factors }\end{array}$} \\
\hline Traditional risk factors & Genetic risk factors & & \\
\hline Cardiac function & Gene polymorphisms & Anesthetic management & Follow-up frequency \\
\hline Pulmonary function & Transcriptional levels & Technical issues & Pharmacologic therapy \\
\hline Renal function & Protein polymorphisms & Duration of the operation & Readmission \\
\hline Age & Protein levels & Completeness-successful & Re-intervention \\
\hline
\end{tabular}

postoperative complications usually occur during hospital stay and rarely during early postdischarge period, and disease recurrence and quality of life can be assessed after discharge from the hospital.

Finally, it is important to mention potential risk factors that the risk stratification models should take into consideration (Table 2). At present, risk stratification models include demographic and traditional risk factors primarily related to cardiac, pulmonary, and renal function, as well as smoking, diabetes, and peripheral vascular disease. In elective surgical procedures, these preoperative characteristics are available and can be used for the estimation of in-hospital mortality risk or major postoperative complications in some other algorithms. In addition to the preoperative traditional risk factors, there are also intraoperative factors (ie, anesthetic management, completeness of tumor and lymph node resection, and duration of the operation) that also affect early and longterm outcome. Indeed, some risk factors acting during the midterm or long-term postoperative period (ie, conservative therapy, follow-up visits, and reinterventions) could also represent important independent predictors of long-term outcome. It is, therefore, obvious that risk stratification begins with the evaluation of preoperative risk factors, but it can also be reconsidered including intraoperative and postoperative risk factors affecting long-term outcome. Moreover, in the era of molecular biology, future risk stratification models face the challenge to include in their algorithms genetic risk factors. It is expected that the inclusion of specific gene and protein polymorphisms, as well as the levels of normal proteins related to the disease, will further increase the discriminatory ability of the risk stratification models. Despite the substantial progress in risk stratification modeling in cardiac surgery, there is no risk stratification model for individual prediction, and as a consequence all algorithms can be used for the estimation of the risk for the outcome used. Similarly, risk stratification models in thoracic surgery are expected to be used for the estimation of the risk for which have been developed. Genetic risk factors may be the key element for achieving individual prediction, but much more work needs to be done, at the moment, for the identification of the genes and proteins, which are associated with thoracic surgical diseases. We have also proposed the development of risk stratification models for the assessment of quality of life after surgery. In this aspect, psychosocial risk factors (ie, anxiety and depression) should be also taken into consideration.

In conclusion, all the above show clearly that risk stratification in thoracic surgery is in its early stages and many studies are needed to reach the progress that has already been made in the field of cardiac surgery. Given the successful story of the STS Adult Cardiac Surgery Database, we are looking forward to seeing the first risk stratification algorithm from the STS General Thoracic Surgery Database, which will also be a good standard for comparison with the Thoracoscore. The real challenge, however, is to develop a risk stratification model including traditional risk factors along with genetic and psychosocial risk factors with respect to long-term outcome to improve the quality of life of our patients and be in accordance with Hippocrates: "to help, or at least do no harm."

\section{References}

1. Falcoz PE, Conti M, Brouchet L, Chocron S, Puyraveau M, Mercier M, et al. The Thoracic Surgery Scoring System (Thoracoscore): risk model for in-hospital death in 15,183 patients requiring thoracic surgery. $J$ Thorac Cardiovasc Surg. 2007;133:325-32.

2. Wright CD, Edwards FH. The Society of Thoracic Surgeons General Thoracic Surgery Database. Ann Thorac Surg. 2007;83:893-4.

3. Chamogeorgakis TP, Connery CP, Bhora F, Nabong A, Toumpoulis IK. Thoracoscore predicts midterm mortality in patients undergoing thoracic surgery. J Thorac Cardiovasc Surg. 2007;134:883-7.

4. Toumpoulis IK, Anagnostopoulos CE, Derose JJ, Swistel DG. European system for cardiac operative risk evaluation predicts long-term survival in patients with coronary artery bypass grafting. Eur J Cardiothorac Surg. 2004;25:51-8.

5. Toumpoulis IK, Anagnostopoulos CE, Toumpoulis SK, DeRose JJ, Swistel DG. EuroSCORE predicts long-term mortality after heart valve surgery. Ann Thorac Surg. 2005;79:1902-8.

6. Toumpoulis IK, Anagnostopoulos CE, Swistel DG, DeRose JJ. Does EuroSCORE predict length of stay and specific postoperative complications after cardiac surgery? Eur J Cardiothorac Surg. 2005;27: 128-33.

7. Toumpoulis IK, Anagnostopoulos CE, Ioannidis JP, Toumpoulis SK, Chamogeorgakis T, Swistel DG, et al. The importance of independent risk-factors for long-term mortality prediction after cardiac surgery. Eur J Clin Invest. 2006;36:599-607.

8. Williams BC, Demitrack LB, Fries BE. The accuracy of the National Death Index when personal identifiers other than Social Security number are used. Am J Public Health. 1992;82:1145-7.

9. Toumpoulis IK, Anagnostopoulos CE. Can EuroSCORE accurately predict long-term outcome after cardiac surgery? Nat Clin Pract Cardiovasc Med. 2005;2:620-1.

10. Chamogeorgakis T, Anagnostopoulos CE, Connery CP, Ashton RC, Dosios T, Kostopanagiotou G, et al. Independent predictors for early and midterm mortality after thoracic surgery. Thorac Cardiovasc Surg. 2007;55:380-4. 